\title{
AVALIAÇÃO MORFOMÉTRICA OVARIANA E RECUPERAÇÃO DE COMPLEXOS CumUlus OOphorus DE FÊMEAS SUÍNAS PRÉ-PÚBERES POR ASPIRAÇÃO FOLICULAR E SECÇÃO DOS OVÁRIOS
}

\author{
(Ovarian morphometric evaluation and recovery of Cumulus-oocyte complexes from \\ prepubertal females swine by follicular aspiration and ovarian slicing)
}

\author{
Andressa Carvalho Neves ${ }^{1}$, Gabrielle Marcondes ${ }^{2}$, Ingrid Caroline da Silva ${ }^{2}$, Maria Marta Loddi ${ }^{3}$, Luciana \\ da Silva Leal Karolewski \\ ${ }^{1}$ Castrolanda, ${ }^{2}$ Curso de Zootecnia, Universidade Estadual de Ponta Grossa, ${ }^{3}$ Departamento de \\ Zootecnia, Universidade Estadual de Ponta Grossa
}

17Corresponding author: andressa_neves@castrolanda.coop.br

RESUMO: Os objetivos do estudo foram caracterizar as dimensões ovarianas e avaliar duas técnicas de recuperação de CCOs (complexos Cumulus oophorus) de ovários de fêmeas suínas pré-púberes, com idade média de cinco meses e peso corporal médio de $80 \mathrm{~kg}$. Os ovários derivados de abatedouro $(\mathrm{n}=127)$ foram pesados usando uma balança digital e medidos (comprimento, largura e altura) com um paquímetro. Dos 127 ovários, 38 foram submetidos à aspiração folicular e 20 foram seccionados utilizando-se uma lâmina de bisturi. Os CCOs dos 58 ovários foram classificados em graus 1, 2, 3 e 4, com o auxílio de um estereomicroscópio. As médias de CCOs recuperados segundo a técnica foram comparadas pelo teste $t$ $(P<0,05)$. As variáveis foram correlacionadas pelo teste de Correlação de Pearson. Os resultados médios observados foram: peso dos ovários 4,26 g; comprimento $2,57 \mathrm{~cm}$; largura $1,99 \mathrm{~cm}$ e altura $1,39 \mathrm{~cm}$. No total, foram recuperados $5.871 \mathrm{CCOs}$ dos 58 ovários. As porcentagens de cada grau foram as seguintes: 0,87\%; 2,23\%; $14,50 \%$ e $82,40 \%$ (graus 1, 2, 3 e 4 , respectivamente). Do número total de CCOs colhidos, o grau 4 foi o mais prevalente e significativo para este experimento. $O$ peso corporal e a largura ovariana exibem maior influência na quantidade e na qualidade dos $\mathrm{CCOs}$. Conclui-se que a técnica de secção provê maior recuperação de CCOs. Quando a técnica de recuperação de CCOs é utilizada em fêmeas suínas pré-púberes prevalece a recuperação de CCOs grau 4.

Palavras-chave: biometria; folículos; marrãs; ovário; oócito.

ABSTRACT: The objectives of the study were to characterize the ovarian dimensions and evaluate two techniques of recovering COC's (Cumulus-oocyte complexes) from the ovaries of gilts with an average age of five months and average body weight of $80 \mathrm{~kg}$. Ovaries from slaughterhouse were used $(n=127)$, which were weighed using a digital balance and measured (length, width and height) with a caliper. Of the 127 ovaries, 38 were submitted to follicular aspiration and 20 were sectioned using a scalpel. The COC's of those 58 ovaries were classified with grades 1, 2, 3 and 4 using a stereomicroscope. The means of COC's recovered according to those techniques were compared by $t$ test $(P<0.05)$. The variables were correlated using the Pearson Correlation test. The average results observed were: ovarian weight $4.26 \mathrm{~g}$; length $2.57 \mathrm{~cm}$; width $1.99 \mathrm{~cm}$ and height $1.39 \mathrm{~cm}$. A total of 5,871 COC's were recovered from the 58 ovaries. The percentages of each grade were as follows: $0.87 \% ; 2.23 \% ; 14.50 \%$ and $82.40 \%$ (grades $1,2,3$ and 4 respectively). Of the total number of COC's collected, grade 4 was the most

Recebido em 12/03/2018

Aprovado em 02/07/2019 
prevalent and significant to this experiment. Body weight and ovarian width have more influence on the quantity and quality of COC's. It is concluded that the ovarian section method provides greater recovery of COC's. When the technique of recovering COC's is used in prepubertal swine females, the recovery of grade 4 COC's prevails.

Keywords: biometry; follicles; gilts; ovary; oocyte. 


\section{INTRODUÇÃO}

Para que as fêmeas suínas alcancem o máximo desempenho reprodutivo é desejável que atinjam idade (seis a sete meses) e peso corporal (120 a $140 \mathrm{~kg}$ ) adequados no início da fase reprodutiva; sendo assim, apresentarão boa taxa de ovulação pela ação de hormônios, com oócitos viáveis para serem fertilizados (Stancic et al., 2012).

Depois de instituída a ciclicidade estral, as porcas apresentam vários cios durante todo o ano, ou seja, são denominadas como poliéstricas anuais. A ausência de cios só ocorre nas fases de gestação e de aleitamento (Soede et al., 2011). O ciclo estral dura em média 21 dias, podendo variar de 18 a 24 dias (Cortez e Toniolli, 2012). As fêmeas exibem também a característica de serem multíparas, pois ocorre a parição de mais de um concepto por gestação, já que ovulam de 15 a 30 oócitos em um período estral (Soede et al., 2011).

Os ovários na espécie suína são constituídos por um corpo maciço e oval, alocando-se próximos aos cornos uterinos (Dyce et al., 2004). Possuem ainda os ovários móveis e irregulares, com os folículos e os corpos lúteos projetados em sua superfície confiando um aspecto lobulado (Sisson, 1986).

O folículo ovariano é a unidade funcional da gônada feminina (Ariyaratna e Gunawardana, 1997). É uma estrutura extremamente organizada, composta basicamente pelo oócito contornado por células foliculares e delimitado por uma membrana basal que o aparta do estroma ovariano (Landim-Alvarenga, 2006). Os folículos são classificados de acordo com o tamanho, tipo, quantidade e morfologia das células da granulosa, podendo ser divididos em pré-antrais e antrais (Landim-Alvarenga, 2006; Paulini, 2014). Os pré-antrais compreendem os folículos primordiais, primários e secundários. Já os folículos antrais abrangem as fases de folículos terciários e pré-ovulatórios (Van den Hurk e Zhao, 2005).

Os folículos são regulados pelas gonadotrofinas até que concluam seu desenvolvimento e seja desencadeada a ovulação. Tais hormônios são provenientes do sistema porta hipotalâmico-hipofisário, sendo que o hipotálamo secreta o GnRH (hormônio liberador de gonadotrofinas) que atua na liberação de $\mathrm{FSH}$ (hormônio folículo estimulante) e LH (hormônio luteinizante) pela hipófise anterior, estabelecendo-se como os hormônios responsáveis pelo desenvolvimento dos folículos, secreção de estrogênio e a ovulação, respectivamente (Eiler, 2007). O fenômeno da ovulação é caracterizado pela liberação do oócito juntamente com o complexo Cumulus oophurus - CCO (Paulini, 2014).

Nas fêmeas suínas, pré e póspúberes, foram descritas as seguintes técnicas para a recuperação de CCOs em laboratório: aspiração folicular (Braga et al., 2007; Grabowska et al., 2016; Yuan et al., 2017), secção/fatiamento do tecido ovariano (Marques et al., 2015) e raspagem da superfície interna dos folículos ovarianos antrais com um par de fórceps (Ikeda e Takahashi, 2003). Destas, as técnicas de aspiração folicular e secção/fatiamento do tecido ovariano foram confrontadas pela primeira vez por Marques et al. (2015) na espécie suína. $\mathrm{Na}$ comparação dos dados, o método de secção/fatiamento rendeu um maior número de CCOs por ovário.

Após a recuperação, deve-se analisar a morfologia dos CCOs pela observação da quantidade e compactação das células da granulosa e homogeneidade do citoplasma do oócito. Sendo assim, são classificados em quatro categorias (graus 1 a 4): sendo que os CCOs identificados como 
grau 1 são os que apresentam maior capacidade para o desenvolvimento embrionário, já os categorizados como grau 4, são menos favoráveis para a produção in vitro de embriões (PIV) suínos e demais espécies (Stojkovic et al., 2001).

Diante do exposto, é apropriado afirmar que pesquisas que investigam a técnica de recuperação dos CCOs suínos com rendimento mais satisfatório podem fornecer dados e um suporte relevante para ensaios futuros. Os objetivos do presente estudo foram: caracterizar as dimensões ovarianas, avaliar a recuperação de CCOs a partir de duas técnicas - aspiração folicular e secção/fatiamento dos ovários e estudar a relação da morfometria ovariana com a recuperação de CCOs em fêmeas suínas pré-púberes.

\section{MATERIAL E MÉTODOS}

O experimento foi aprovado pela Comissão de Ética no Uso de Animais (CEUA) da Universidade Estadual de Ponta Grossa (UEPG)/PR, Brasil, registrado no Processo $n^{\circ}$ 016/2016 desta Comissão.

Os ovários foram obtidos no abatedouro Luiz Antônio, localizado na cidade de Ponta Grossa/PR, e processados no laboratório de Anatomia e Reprodução Animal da UEPG, durante o período de julho a setembro de 2016.

Os ovários eram provenientes de fêmeas suínas pré-púberes, sendo os mais facilmente disponibilizados, pois a maioria das fêmeas abatidas nos frigoríficos para produção comercial de carne é composta por fêmeas jovens que ainda não ciclaram. Os ovários prépúberes foram confirmados pela ausência de corpos lúteos. A idade média das fêmeas era de cinco meses e o peso corporal médio de $80 \mathrm{~kg}$. Após a colheita, os ovários foram higienizados e acondicionados em recipientes plásticos com tampa, preenchidos com solução fisiológica a $0,9 \%$ de $\mathrm{NaCl}$, aquecida a $39^{\circ} \mathrm{C}$. Para manter a temperatura, os ovários foram transportados dentro de uma caixa de isopor até o laboratório.

No laboratório, os ovários foram realocados em béqueres de vidro, onde permaneceram aquecidos a $39^{\circ} \mathrm{C}$ em banho maria (modelo: TE-056 MAG, $\mathrm{TECNAL}^{\circledR}$ ). Cada ovário foi dissecado com o auxílio de tesoura e pinça anatômica autoclavadas e, a seguir, foram feitas as seguintes avaliações: peso $(\mathrm{g})$, comprimento $(\mathrm{cm})$, largura $(\mathrm{cm})$ e altura $(\mathrm{cm})$ do ovário. O peso do ovário foi determinado em balança digital (modelo: BL320H, MARTE ${ }^{\circledR}$ ). As medidas dos ovários foram realizadas com o uso de um paquímetro, com escala de 0,05 mm (DISMA $\left.{ }^{\circledR}\right)$.

Foram empregadas duas técnicas de recuperação dos CCOs, sendo estas: a aspiração folicular (Figura 1a) e a secção/fatiamento dos ovários (Figura 1b). Para a técnica de aspiração, utilizou-se agulha $40 \times 12 \mathrm{~mm}$ acoplada à seringa de $10 \mathrm{~mL}$. Foram aspirados os folículos ovarianos antrais para a obtenção do líquido folicular, que foi transferido para uma placa de Petri para posterior contagem e classificação dos CCOs.

Já para a técnica de secção/fatiamento dos ovários, os mesmos foram seccionados com 0 auxílio de uma lâmina de bisturi, realizando vários cortes para o rompimento dos folículos ovarianos, sobre placas de Petri preenchidas com solução fisiológica.

Os CCOs foram avaliados em estereomicroscópios $\left(\mathrm{OPTECH} \mathrm{H}^{\circledR}\right)$ e classificados em graus 1, 2, 3 e 4 conforme proposto por Stojkovic et al. (2001): grau 1 - oócito com citoplasma homogêneo e granulações finas e múltiplas camadas compactas de células do Cumulus (Figura 2a); grau 2 oócito com pequenas áreas de 
pigmentação, com pelo menos cinco camadas completas de células ao redor, porém menos compactas (Figura 2b); grau 3 - citoplasma do oócito heterogêneo/vacuolizado, coberto com três camadas de células e/ou pequenas áreas desnudas (Figura 2c) e grau 4 oócito heterogeneamente pigmentado e camadas celulares do Cumulus ausentes ou expandidas (Figura 2d).
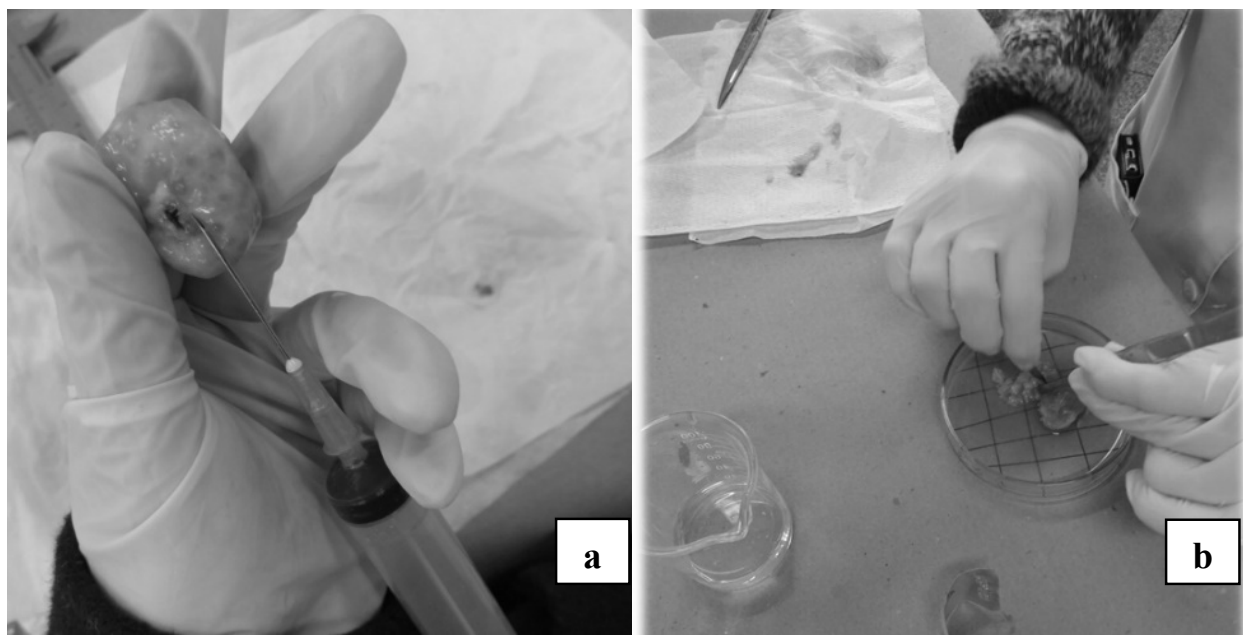

Figura 1 - a) Aspiração dos folículos ovarianos; b) Secção dos ovários de fêmeas suínas.

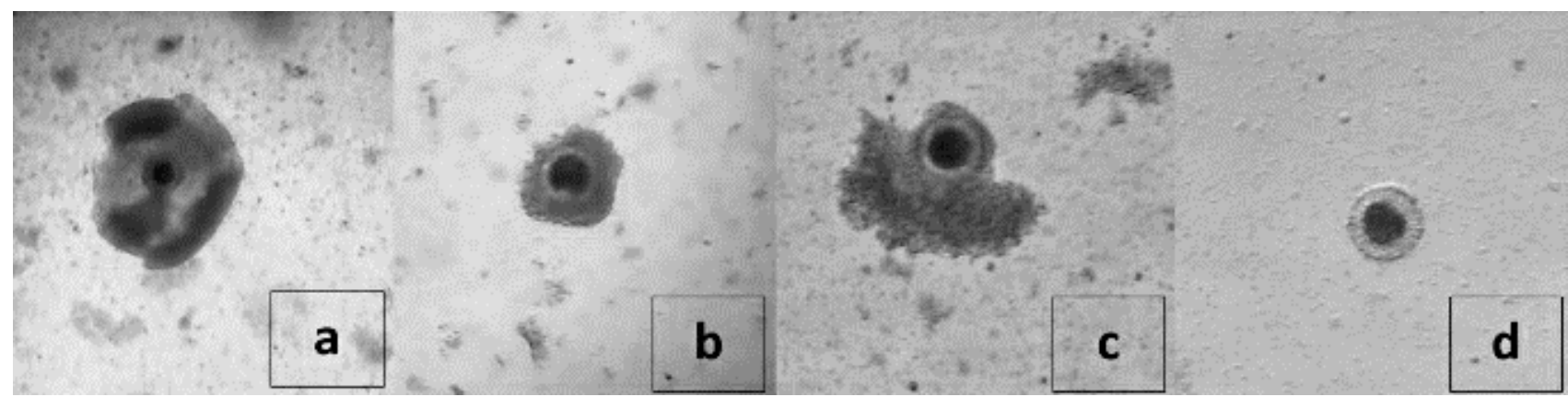

Figura 2 - Classificação dos Complexos Cumulus oophorus: a) CCO grau 1; b) CCO grau 2; c) CCO grau 3; d) CCO grau 4.

O delineamento foi inteiramente casualizado, ou seja, os ovários de cada técnica de recuperação foram escolhidos aleatoriamente. O software estatístico utilizado para a análise estatística dos resultados foi o Minitab 17.3.1 (2016). Os dados de biometria ovariana ( $n=127 \quad$ ovários $)$ foram analisados por meio de estatística descritiva (média e erro-padrão da média). Os valores médios de quantidade e qualidade dos $\operatorname{cCOs}$ obtidos segundo a técnica de recuperação $\quad(n=58 ; 38$ ovários aspirados e 20 ovários seccionados) foram submetidos ao teste $t$ para duas amostras. Os dados de peso e medidas ovarianas, assim como número e grau dos CCOs ( $\mathrm{n}=58$ ovários) foram correlacionados entre si pelo teste de Correlação de Pearson, sendo considerada uma correlação muito fraca $r \leq 0,25$, fraca $r>0,25$ e $\leq 0,50$, moderada $r>0,50$ e $\leq 0,75$ e forte quando $r>0,75$. $\mathrm{Em}$ todas as análises, os efeitos foram declarados significativos quando $\mathrm{P}<0,05$. 


\section{RESULTADOS E DISCUSSÃO}

A tabela 1 apresenta o peso e a morfometria ovariana de 127 ovários de fêmeas suínas pré-púberes. Oberlender et al. (2014) e Natal et al. (2016) identificaram pesos médios de ovários de marrãs pré-púberes variando de 3 a $4,7 \mathrm{~g}$ e 3,82 a 4,32 g respectivamente, dados que são concordantes com o encontrado no presente estudo para fêmeas em idade similar.
O comprimento e a largura médios dos ovários aproximaram-se aos relatados por Alves (2010) que foram $2,50 \pm 0,32$ e $1,55 \pm 0,22 \mathrm{~cm}$, respectivamente. Já Vexenat (2012) obteve valor médio de $1,15 \mathrm{~cm}$ para altura de ovários de fêmeas suínas, com idade média de cinco meses, valor próximo ao identificado no presente estudo.

Tabela 1 - Valores médios e erro padrão da média (EPM) do peso e das medidas ovarianas de 127 ovários de fêmeas suínas pré-púberes.

\begin{tabular}{c|c|c}
\hline Características Ovarianas & $\mathrm{N}$ & Média \pm EPM \\
\hline Peso $(\mathrm{g})$ & 127 & $4,26 \pm 0,01$ \\
Comprimento $(\mathrm{cm})$ & 127 & $2,57 \pm 0,03$ \\
Largura $(\mathrm{cm})$ & 127 & $1,99 \pm 0,03$ \\
Altura $(\mathrm{cm})$ & 127 & $1,39 \pm 0,02$ \\
\hline
\end{tabular}

Muitos fatores podem influenciar a morfometria ovariana, como por exemplo, a espécie estudada, a fase do ciclo estral, a genética, o peso corporal, a nutrição, o efeito-macho e também a idade da fêmea (Priedkalns e Leiser, 2006).

Devido à interferência de múltiplos fatores nas dimensões ovarianas, a comparação dos dados na literatura torna-se difícil, principalmente se for efetuada com publicações mais antigas, nas quais é mais fácil encontrar as medidas anatômicas dos genitais. Atualmente, os animais apresentam potencial genético para crescimento corporal diferenciado e mais avançado em relação a tempos passados, com isso atingem a puberdade antecipadamente devido à heterose proveniente de cruzamentos com raças mais precoces (Monteiro, 1999) e isso pode representar medidas ovarianas diferentes.

Como citado anteriormente, a idade também é um elemento importante que afeta a atividade ovariana, resultando em proporções ovarianas distintas. Fêmeas suínas mais velhas (24 a 84 meses de idade) possuem pesos ovarianos maiores do que fêmeas de cinco meses de idade (Vexenat, 2012). A mesma ocorrência foi verificada na espécie bovina, evidenciando que novilhas pré-púberes apresentam pesos ovarianos menores (Nascimento et al., 2003).

$\mathrm{Na}$ tabela 2, encontram-se os valores médios das propriedades ovarianas avaliadas de acordo com a técnica de recuperação de CCOs, ou seja, aspiração ou secção/fatiamento dos ovários.

No total, foram recuperados 5.871 CCOs, sendo eles $0,87 \%$ grau $1(n=51)$; $2,23 \%$ grau $2(n=131) ; 14,50 \%$ grau 3 $(n=850)$ e por fim $82,40 \%$ classificados como grau 4 ( $n=4.839)$. Constatou-se que não houve efeito da técnica na recuperação de CCOs graus 1 e 3 $(P>0,05)$. No entanto, com relação aos CCOs grau 4, a secção ovariana 
resultou em média superior à aspiração folicular $(P<0,05)$. Em contrapartida, a aspiração folicular conferiu maior quantidade de CCOs grau 2. Houve ainda diferença significativa para o total de CCOs, que foi superior para o procedimento de secção ovariana. É importante ressaltar que as dimensões ovarianas foram consideradas como covariáveis na análise estatística, para que a superioridade nas medidas de largura e altura dos ovários submetidos ao fatiamento não interferisse nos resultados de recuperação dos CCOs.

Tabela 2 - Valores médios de peso, medidas ovarianas e números de complexos Cumulus oophorus de graus 1, 2, 3, 4 e total recuperado de ovários de fêmeas suínas pré-púberes, segundo a técnica de recuperação.

\begin{tabular}{c|cccc}
\hline \multicolumn{4}{c}{ Técnica de Recuperação } \\
\hline Variáveis & Aspiração $(\mathrm{n}=38)$ & Secção/fatiamento $(\mathrm{n}=$ & $\mathrm{P}$ \\
\hline Peso do ovário $(\mathrm{g})$ & 4,31 & 4,80 & 0,16 \\
Comprimento $(\mathrm{cm})$ & 2,49 & 2,69 & 0,11 \\
Largura $(\mathrm{cm})$ & $1,89 \mathrm{~b}^{*}$ & $2,34 \mathrm{a}$ & 0,00 \\
Altura $(\mathrm{cm})$ & $1,30 \mathrm{~b}$ & $1,59 \mathrm{a}$ & 0,00 \\
CCOs G1 & 0,87 & 0,90 & 0,92 \\
CCOs G2 $^{2}$ & $2,74 \mathrm{a}$ & $1,35 \mathrm{~b}$ & 0,01 \\
CCOs G3 $^{3}$ & 12,11 & 19,50 & 0,13 \\
CCOs G4 $^{4}$ & $24,60 \mathrm{~b}$ & $195,20 \mathrm{a}$ & 0,00 \\
Total CCOs $^{5}$ & $40,50 \mathrm{~b}$ & $216,90 \mathrm{a}$ & 0,00
\end{tabular}

${ }^{1} \mathrm{CCOs}$ G1- complexos Cumulus oophorus grau 1; ${ }^{2} \mathrm{CCOs}$ G2- complexos Cumulus oophorus grau 2; ${ }^{3} \mathrm{CCOs}$ G3- complexos Cumulus oophorus grau $3 ;{ }^{4} \mathrm{CCOs}$ G4- complexos Cumulus oophorus grau 4; ${ }^{5}$ Total CCOs- número total de complexos Cumulus oophorus.

*Médias que não compartilham a mesma letra indicam diferença entre as médias $(P<0,05)$.

Marques et al. (2015), estudando diferentes técnicas de recuperação de CCOs em ovários de fêmeas suínas, observaram uma maior aquisição de CCOs de grau 1 por intermédio da técnica de secção/fatiamento quando comparada com a aspiração dos folículos, o que divergiu dos dados encontrados no presente estudo, já que não houve diferença significativa entre as técnicas para a recuperação de complexos de grau 1. Em outra pesquisa realizada com ovários de fêmeas caprinas, Ambili et al. (2015) registraram uma maior recuperação de CCOs/ovário na técnica de secção/fatiamento em relação à punção e aspiração do tecido ovariano, do mesmo modo que foi constatado no presente estudo, quando as técnicas foram confrontadas. A mesma diferença nesse parâmetro foi observada por Santos et al. (2016) em ovários de fêmeas bovinas post mortem (14,8 vs.
7,5 oócitos/ovário para fatiamento e aspiração folicular, respectivamente).

A maior obtenção de CCOs se deve ao fato de que o fatiamento confere uma maior superfície de exposição do ovário, ou seja, havia a possibilidade de romper mais folículos. Em adição, os cortes do tecido ovariano permitem a colheita de oócitos presentes em folículos pequenos, que normalmente não são aspirados. A utilização da técnica de secção/fatiamento pode ser proveitosa quando se almeja obter uma alta quantidade de CCOs, principalmente em situações de pouca disponibilidade de ovários (Santos et al., 2016). No caso do presente estudo, apesar da vantagem numérica, a técnica de secção/fatiamento resultou em um maior número de CCOs grau 4, o que não é desejável para estudos com PIV.

A baixa recuperação de $\mathrm{CCO}$ de grau 1 pode ser explicada pela idade 
das fêmeas (apenas cinco meses). A maioria das fêmeas utilizadas em pesquisas para aspiração dos folículos é pré-púbere e isso torna a técnica mais trabalhosa resultando em menores taxas de recuperação de oócitos (Marques et al., 2015). De acordo com Grabowska et al. (2016), fêmeas suínas pré-púberes apresentam oócitos com menor diâmetro e citoplasma em desenvolvimento e imaturos, em relação aos oócitos de fêmeas adultas; deste modo pode-se haver correlação entre a idade dos animais (maturidade sexual) com o tamanho e a qualidade dos oócitos.

$\mathrm{Na}$ tabela 3 , estão representadas as correlações simples entre as características ovarianas, o número e a classificação de CCOs dos 58 ovários.

Conforme observado na tabela 3 , o peso corporal e a largura ovariana manifestaram uma influência mais marcante do que o peso do ovário e as outras dimensões ovarianas na quantidade de CCOs.

Tabela 3 - Estudo da correlação entre as variáveis peso corporal do animal (kg) e peso (g), comprimento $(\mathrm{cm})$, largura $(\mathrm{cm})$, altura $(\mathrm{cm})$ e recuperação de complexos Cumulus oophorus grau 1 , 2, 3 e 4 de ovários de fêmeas suínas pré-púberes.

\begin{tabular}{c|c|c|c|c|c|c} 
Variáveis & $\begin{array}{c}\text { Peso } \\
\text { corporal } \\
(\mathrm{kg})\end{array}$ & ccos G1 & ccos G2 & CcOs G3 & CcOs G4 & $\begin{array}{c}\text { Total } \\
\text { CcOs }^{5}\end{array}$ \\
\hline $\begin{array}{c}\text { Peso corporal (kg) } \\
\text { Peso do }\end{array}$ & 1,00 & $-0,00$ & $-0,26^{*}$ & 0,18 & $0,64^{*}$ & $0,62^{*}$ \\
$\begin{array}{c}\text { ovário (g) } \\
\text { Comprimento (cm) }\end{array}$ & $0,34^{*}$ & 0,05 & 0,13 & $0,36^{*}$ & $0,31^{*}$ & $0,35^{*}$ \\
Largura (cm) & $0,31^{*}$ & 0,21 & 0,07 & $0,38^{*}$ & $0,42^{*}$ & $0,46^{*}$ \\
Altura (cm) & $0,51^{*}$ & 0,01 & $-0,12$ & $0,36^{*}$ & $0,61^{*}$ & $0,63^{*}$ \\
Total Ccos & $0,62^{*}$ & 0,07 & $-0,21$ & $0,43^{*}$ & $0,99^{*}$ & 1,00
\end{tabular}

${ }^{1} \mathrm{CCOs}$ G1- complexos Cumulus oophorus grau 1; ${ }^{2} \mathrm{CCOs}$ G2- complexos Cumulus oophorus grau 2; ${ }^{3} \mathrm{CCOs}$ G3- complexos Cumulus oophorus grau $3 ;{ }^{4} \mathrm{CCOs}$ G4- complexos Cumulus oophorus grau $4 ;{ }^{5}$ Total CCOs- número total de complexos Cumulus oophorus. ${ }^{*} \mathrm{P}<0,05$.

De forma geral, o total de CCOs sofreu interferência (fraca a moderada) das dimensões ovarianas. Isso significa que, quanto mais pesado e maior o ovário, mais expressiva foi a obtenção de CCOs, pois essas características representavam uma superioridade na extensão do parênquima ovariano. Essa mesma influência foi verificada nos CCOs de grau 4, uma vez que esta categoria foi a mais abundantemente recuperada (correlação de 0,99 em relação ao total de CCOs).

\section{CONCLUSÕES}

O estudo descreve a morfometria ovariana de fêmeas suínas pré-púberes, cujos dados se assemelham aos registros encontrados na literatura em fêmeas da mesma idade. A técnica de secção/fatiamento dos ovários suínos resulta em maior recuperação de CCOs. Quando a técnica de recuperação de CCOs é utilizada em fêmeas suínas prépúberes prevalece a recuperação de oócitos grau 4. O peso corporal e a largura do ovário são as variáveis que mais interferem no número e na qualidade dos CCOs.

\section{AGRADECIMENTOS}

Os autores agradecem à Fundação Araucária pela bolsa de 
estudo concedida e ao abatedouro Luiz Antônio por ceder o material de estudo.

\section{REFERÊNCIAS}

ALVES, B.G. Isolamento, quantificação e classificação de folículos pré-antrais de suínos. 2010. Uberlândia, 52 f. Dissertação (Mestrado), Universidade Federal de Uberlândia.

AMBILI, J.; JOSEPH, M.; VIJAYAKUMARAN, V. Effect of oocyte retrieval techniques on yield and quality of caprine oocytes. Journal of Agriculture and Veterinary Science, $v$. 8, n. 4, p. 50-52, 2015. DOI: 10.9790/2380-08415052.

ARIYARATNA,

H.B.S.; GUNAWARDANA, V.K. Morphology and morphometry of ovarian follicles in the goat. Small Ruminant Research, v. 26, n. 1-2, p. 123-129, 1997 . DOI: 10.1016/s0921-4488(96)00995-9.

BRAGA, D.P.A.F.; PASQUALOTTO, F,F.; MADASCHI, C. et al. Use of pig oocytes for training new professionals in human assisted reproduction laboratories. Fertility and Sterility, v. 88 , n.5, p. 1408-1412, 2007. DOI: 10.1016/j.fertnstert.2007.01.017.

CORTEZ, A.A; TONIOLLI, R. Aspectos fisiológicos e hormonais da foliculogênese e ovulação em suínos. Revista Brasileira de Reprodução Animal, v. 36, n. 3, p 163-173, 2012. Disponível em: <http://cbra.org.br/pages/publicacoes/rbr a/v36n3/p163-173\%20(RB240).pdf>. Acesso: 6/11/2018.

DYCE, K.M.; SACK, W.O.; WENSING, C.J.G. Órgãos reprodutivos femininos: Ovários. In: Tratado de Anatomia Veterinária. 3. ed. Rio de Janeiro: Elsevier, 2004, p. 191-192.

EILER, H. Glândulas endócrinas. In: REECE, W.O. Dukes fisiologia dos animais domésticos. 12. ed. Rio de
Janeiro: Guananabara Koogan, 2007, Cap. 37, p. 577-622.

GRABOWSKA, R.; BLASZCZYK, B.; STANKIEWICZ, T. et al. Quality of oocytes in prepubertal and pubertal swine. Turkish Journal of Veterinary and Animal Sciences, v. 40, p 89-94, 2016. DOI: 10.3906/vet-1502-16.

IKEDA, K.; TAKAHASHI, Y. Comparison of a maturational and developmental parameters of oocytes recovered from prepubertal and adult pigs. Reproduction, Fertility and Development, v. 15, p. 215-221, 2013. DOI: 10.1071/RD02077.

LANDIM-ALVARENGA, F.C. Fecundação e clivagem. In: PRESTES, N.C.; LANDIM-ALVARENGA, F.C. Obstetrícia Veterinária. Rio de Janeiro: Guanabara Koogan, 2006, Cap. 1, p. 121.

MARQUES, M.G; BARROS, F.R.O. de; GOISSIS, M.D. et al. Effect of oocyte recovery techniques on in vitro production of swine embryos. Open Journal of Animal Sciences, v. 5, n. 4, p. 467-473, 2015. DOI:10.4236/ojas.2015.54048.

MINITAB. Minitab Inc. Versão 17, 2016. MONTEIRO, M.H.G.V. Aspectos reprodutivos de fêmeas da raça suína alentejana. 1999. Lisboa, $107 f$. Dissertação (Mestrado). Universidade Técnica de Lisboa.

NASCIMENTO, A.A. do; PINHEIRO, N.L; SALES, A. et al. Correlação morfométrica do ovário de fêmeas bovinas em diferentes estágios reprodutivos. Brazilian Journal of Veterinary Research and Animal Science, v. 40, n. 2, p.126-132, 2003. DOI: $10.1590 / \mathrm{S} 1413-$ 95962003000200006.

NATAL, F.L.N; MELO, A.J.F. de; JIMENEZ, D.L.F. et al. Indução da ovulação e características ovarianas de 
marrãs pré-púberes tratadas com gonadotrofina coriônica humana ou kisspeptina. Boletim de Indústria Animal, v.73, n.1, p.9-14, 2016. DOI: 10.17523/bia.v73n1p9.

OBERLENDER, G.; PONTELO, T. P.; MIRANDA, J. R. et al. Morphological and morphometric evaluation of prepubertal gilt ovaries, uterine tubes and uterus at different oestrus cycle stages. Pesquisa Veterinária Brasileira, v. 34, n. 1, p.83-90, 2014. DOI: $10.1590 / \mathrm{s} 0100$

736x2014000100014.

PAULINI, F. Técnicas de imagem no estudo comparativo de ovários de diferentes espécies domésticas. 2014. Brasília, 98f. Tese (Doutorado), Universidade de Brasília.

PRIEDKALNS, J.; LEISER, R. Female reproductive system. In: EURELL, J.A.; FRAPPIER, B. L. Dellmann's Textbook of Veterinary Histology. 6. ed. Ames: Blackwell Publishing, 2006, p. 256-278.

SANTOS, M.V.O.; BORGES, A.A.; QUEIROZ NETO, L.B. et al. Influência do método de recuperação oocitária sobre os parâmetros quanti-qualitativos de oócitos bovinos. Ars Veterinaria, v. 32 , n. 2, p. 105-109, 2016. DOI: 10.15361/2175-0106.2016v32n2p105109.

SOEDE, N.M.; LANGENDIJK, P.; KEMP, B. Reproductive cycle in pigs. Animal Reproduction Science, v. 124, p. 251-258, 2011.2 DOI: 10.1016/j.anireprosci.2011.02.025.

SISSON, S. Sistema urogenital do suíno. In: GETTY, R. Sisson e Grossman anatomia dos animais domésticos. 5. ed. Rio de Janeiro: Guanabara Koogan, 1986, Cap. 42, p.1216-1221.

STANCIC, I.; BOSNJAK, D.; RADOVIC, I. et al. Ovarian reaction and estrus manifestation in delayed puberty gilts after treatment with equine chorionic gonadotropin. Reproductive Biology and Endocrinology, v.10, n. 61, p.1-5, 2012. DOI: 10.1186/1477-7827-10-61.

STOJKOVIC, M; MACHADO, A.S; STOJKOVIC, $P$. et al. Mitochondrial distribution and adenosine triphosphate content of bovine oocytes before and after in vitro maturation: correlation witch morphological criteria and developmental capacity after in vitro fertilization and culture. Biology of Reproduction, v. 64, n. 3, p.904-909, 2001. DOI: 10.1095/biolreprod64.3.904.

VAN DEN HURK, R.; ZHAO J. Formation of mammalian oocytes and their growth, differentiation and maturation within ovarian follicles. Theriogenology, v. 63, n. 6, p17171751, 2005.

DOI: 10.1016/j.theriogenology.2004.08.0 05.

VEXENAT, S.C.O.R. Avaliação endócrina e morfológica do trato genital de fêmeas suínas. 2012. Botucatu, 64 f. Dissertação (Mestrado) Universidade Estadual Paulista.

YUAN, Y.; SPATE, L.D.; REDEL, B. et al. Quadrupling efficiency of genetically modified pigs through improved oocyte maturation. PNAS, p. 5796- 5804, 2017. DOI: 10.1073/pnas.1703998114. 